\title{
Condutas nutricionais no cuidado das manifestações da Síndrome dos Ovários
}

\section{Policísticos}

\author{
Nutritional conduct in the care of Polycystic Ovary Syndrome manifestations \\ Conducta nutricional en el cuidado de las manifestaciones del Síndrome de Ovario Poliquistico
}

Recebido: 02/12/2021 | Revisado: 07/12/2021 | Aceito: 11/12/2021 | Publicado: 20/12/2021

Caroline Maria Vasconcelos Assunção
ORCID: https://orcid.org/0000-0001-8238-2994
Centro Universitário Santo Agostinho, Brasil
E-mail: carolinemvasconcelos8@ gmail.com
Luiza Marly Freitas de Carvalho
ORCID: https://orcid.org/0000-0002-6726-3994
Centro Universitário Santo Agostinho, Brasil
E-mail: lumarnahid@ @mail.com

\begin{abstract}
Resumo
O objetivo desta revisão foi avaliar os efeitos de padrões nutricionais indicados para o manejo da Síndrome dos Ovários Policísticos (SOP). Para tanto, foi realizado uma busca bibliográfica na base de dados PubMed, compreendendo artigos publicados entre 2018 e 2021, em inglês e português. Foi encontrado 1141 artigos, e 610 artigos foram excluídos por não terem relação com o tópico estudado ou por não serem disponibilizados gratuitamente. Dos 531 artigos, 41 foram selecionados e 20 estudos foram incluídos na revisão. Como resultado, os estudos sugerem que intervenções nutricionais, como dietas de baixo teor de carboidratos $(<45 \%)$ e de baixo índice glicêmico, suplementação de ômega 3 e compostos fenólicos, maior ingestão de leguminosas e vegetais, parecem ter efeito benéfico, quando ajustadas à necessidade individual, sobre as manifestações clínicas e bioquímicas em mulheres com SOP. O presente estudo se alinha com as evidências de que uma variedade de condutas dietéticas balanceadas, podem e devem ser recomendadas para o manejo dessas manifestações. No entanto, os princípios gerais de uma alimentação saudável, de acordo com as recomendações para população geral, são cabíveis para todas as mulheres com SOP. Para estas, não há ou há evidências limitadas de que qualquer tipo específico de dieta seja melhor do que outro.
\end{abstract}

Palavras-chave: Síndrome Ovários Policísticos; Efeitos; Dieta; Tratamento; Condutas nutricionais.

\begin{abstract}
The objective of this review was to evaluate the effects of nutritional patterns indicated for the management of Polycystic Ovary Syndrome (PCOS). For this purpose, a bibliographic search was carried out in the PubMed database, comprising articles published between 2018 and 2021, in English and Portuguese. A total of 1141 articles were found, and 610 articles were excluded for being unrelated to the topic studied or for not being freely available. Of the 531 articles, 41 were selected and 20 studies were included in the review. As a result, the studies suggest that nutritional interventions, such as low-carbohydrate $(<45 \%)$ and low-glycemic index diets, supplementation of omega-3 and phenolic compounds, higher ingestion of legumes and vegetables, appear to have a beneficial effect, when adjusted to individual need, on clinical and biochemical manifestations in women with PCOS. The present study is in line with the evidence that a variety of balanced dietary approaches can and should be recommended for the management of these manifestations. However, the general principles of healthy eating, as recommended for the general population, are applicable to all women with PCOS. For these, it is not known so far whether or not there is limited evidence that any specific type of diet is better than another.
\end{abstract}

Keywords: Polycystic Ovary Syndrome; Effects; Diet; Treatment; Nutritional conduct.

\section{Resumen}

El objetivo de esta revisión fue evaluar los efectos de los patrones nutricionales indicados para el tratamiento del SOP. Para ello, se realizó una búsqueda bibliográfica en la base de datos PubMed, compuesta por artículos publicados entre 2018 y 2021, en inglés y portugués. Se encontraron 1141 artículos y se excluyeron 610 artículos por no estar relacionados con el tema estudiado o por no estar disponibles gratuitamente. De los 531 artículos, se seleccionaron 41 y se incluyeron 20 estudios en la revisión. Como resultado, los estudios han demostrado que las intervenciones nutricionales, como las dietas bajas en carbohidratos $(<45 \%)$ y de índice glucémico bajo, la suplementación de omega 3 y compuestos fenólicos, el aumento de la ingesta de legumbres y verduras, parecen tener un efecto beneficioso cuando se ajusta a la necesidad individual, sobre las manifestaciones clínicas y bioquímicas en mujeres con SOP. El presente estudio está en línea con la evidencia de que una variedad de enfoques dietéticos balanceados pueden y deben 
recomendarse para el manejo de las manifestaciones clínicas y metabólicas en mujeres con SOP. Sin embargo, los principios generales de una alimentación saludable, recomendados para la población general, son aplicables a todas las mujeres con SOP. Para estos, no hay evidencia o es limitada de que un tipo específico de dieta sea mejor que otro.

Palabras clave: Síndrome de Ovario Poliquistico; Efectos; Dieta; Tratamiento; Comportamientos nutricionales.

\section{Introdução}

A síndrome dos ovários policísticos (SOP) é uma endocrinopatia comum apresentada por 6-21\% das mulheres em idade reprodutiva (Kite, et al., 2019). Este dado varia em diferentes países, talvez devido a diferença na população em estudo, a variedade de critérios usados para defini-lo, os pontos de corte inconsistentes e o método usado para definir cada critério (Kazemi et al., 2021).

De acordo com os critérios de Rotterdam, o diagnóstico de SOP depende da identificação de pelo menos duas das três características a seguir: oligoanovulação, hiperandrogenismo e ovários policísticos na ultrassonografia (Haidari et al.,2020). As mulheres podem apresentar distúrbios reprodutivos (infertilidade e complicações na gravidez), metabólicos (aumento dos fatores de risco e possível maior prevalência de diminuição da tolerância à glicose, diabetes tipo 2 e doença cardiovascular) e psicológicos (piora da qualidade de vida e aumento da ansiedade e depressão). (Moran et al., 2019)

Atualmente, a etiologia da SOP não é completamente compreendida, e pode representar a consequência da interação entre fatores genéticos e ambientais, incluindo história familiar, baixo peso ao nascer, obesidade, hábitos alimentares inadequados e estilo de vida sedentário (Zhang et al., 2019). A fisiopatologia da SOP está centrada no hiperandrogenismo e na RI e pode causar muitas complicações de curto ou longo prazo, como aborto espontâneo, síndrome metabólica e câncer endometrial (Zhou et al., 2020).

Dentre os mecanismos endócrinos envolvidos na etiopatogênese da SOP está o padrão de secreção de gonadotrofinas alterado, com hipersecreção característica de Hormônio Luteinizante (Lutheinizing Hormone - LH), evento patognomônico desta síndrome, com aumento na amplitude dos pulsos e, a secreção de Hormônio Folículo Estimulante (Follicle Stimulating Hormone - FSH) baixa ou no limite inferior da normalidade. Os baixos níveis de FSH não farão estímulo suficiente para que os folículos primários cheguem ao estágio de folículos maduros, que se acumularão e darão aspecto policístico aos ovários (Febrasgo, 2018).

A adiposidade visceral, comum em mulheres obesas e não obesas com SOP, demonstrou amplificar e piorar o hiperandrogenismo e a RI, o que, por sua vez, induziria o acúmulo de gordura abdominal, formando um ciclo vicioso de feedback. As interações entre androgênio, RI e obesidade afetam profundamente o metabolismo endócrino, levando a distúrbios da ovulação, comprometimento do desenvolvimento potencial do óvulo e baixa receptividade endometrial (Shang et al., 2021).

Mulheres com SOP além da propensão à obesidade, percebem uma inevitabilidade para ganho de peso, exibem baixa adesão a dietas com restrição calórica, e têm tendência para o retorno do peso longitudinal (Kazemi et al., 2021). O fenótipo clínico é ainda piorado como resultado da liberação excessiva de androgênio ovariano e adrenal, bem como globulina de ligação do hormônio sexual hepático (SHBG), cuja secreção é independente da obesidade. (Haudum et al., 2020)

Estudos anteriores revelaram que fatores ambientais podem representar fatores fundamentais na incidência e no tratamento da doença. Entre esses fatores ambientais, os padrões alimentares são considerados um dos mais importantes e controláveis (Salek et al., 2019). Portanto, em busca da compreensão do tipo e composição da dieta mais eficaz para fornecer estratégias de manejo sustentáveis e bem-sucedidos na SOP, o artigo tem como objetivo fazer uma busca na literatura sobre as condutas nutricionais capazes de auxiliar no cuidado, ao longo da vida, desta população clínica. 


\section{Metodologia}

O presente estudo trata-se de uma revisão bibliográfica integrativa, metodologia que integra achados advindos de pesquisas com diferentes desenhos, permitindo a compreensão de problemas relevantes e a definição de novos conceitos (Yonekura et al., 2019). Para contextualizar e apresentar ao leitor as condutas nutricionais no cuidado das manifestações clínicas e metabólicas da Síndrome dos Ovários Policísticos (SOP), foi utilizada a base de dados Pub Med, e os seguintes descritores em inglês foram dispostos: "polycystic ovary syndrome", "nutritional conduct", "diet" "treatment". Com o objetivo de possibilitar uma busca mais assertiva dos artigos, os termos encontrados foram combinados, utilizando o operador booleano AND, para concluir a busca.

Os critérios de inclusão foram artigos entre 2018 e 2021, em português e inglês com disponibilidade gratuita na íntegra, bem como tivesse as palavras "sop" "condutas nutricionais ou "dieta" ou "condutas dietéticas" "suplementos alimentares" nos títulos e resumos. Foi encontrado 1141 artigos, e 610 artigos foram excluídos por não terem relação com o tópico estudado ou por não serem disponibilizados gratuitamente. Dos 531 artigos, 41 foram selecionados e 20 estudos foram incluídos na revisão.

\section{Resultados e Discussão}

Os resultados foram dispostos em tabela, sendo a tabela 1 destinada aos estudos que avaliaram o efeito de intervenções nutricionais em mulheres com SOP.

Tabela 1: Estudos que compararam o efeito de diferentes tipos de intervenção nutricional em mulheres com SOP.

\begin{tabular}{|c|c|c|c|c|c|}
\hline Autor & Título & Tipo de Estudo & $\begin{array}{l}\text { Número de par- } \\
\text { ticipantes }\end{array}$ & Intervenção Nutricional & Resultados \\
\hline $\begin{array}{l}\text { ZHANG et al., } \\
2019\end{array}$ & $\begin{array}{l}\text { O efeito da dieta } \\
\text { pobre em carboidra- } \\
\text { tos na síndrome do } \\
\text { ovário policístico: } \\
\text { uma meta-análise de } \\
\text { ensaios clínicos } \\
\text { randomizados. }\end{array}$ & $\begin{array}{l}\text { Ensaios clínicos } \\
\text { randomizados. }\end{array}$ & 327 & $\begin{array}{l}\text { Dieta pobre em carboidratos, } \\
\text { na qual representaram menos } \\
\text { de } 45 \% \text { dos três principais } \\
\text { nutrientes, e o grupo de con- } \\
\text { trole estava em uma dieta } \\
\text { regular (carboidratos represen- } \\
\text { taram aproximadamente } 45 \% \text { ) }\end{array}$ & $\begin{array}{l}\text { Redução do IMC, HOMA-IR, } \\
\text { TC e LDL-C.Análises estrati- } \\
\text { ficadas indicaram que LCD } \\
\text { com duração superior a } 4 \\
\text { semanas teve um efeito mais } \\
\text { forte no aumento dos níveis } \\
\text { de FSH, aumentando os níveis } \\
\text { de SHBG, e T decrescente. }\end{array}$ \\
\hline $\begin{array}{l}\text { KAZEMI et al., } \\
2018\end{array}$ & $\begin{array}{l}\text { Uma comparação entre uma } \\
\text { dieta baseada no pulso e a dieta } \\
\text { de mudanças terapêuticas no } \\
\text { estilo de vida em combinação } \\
\text { com exercícios e aconselha- } \\
\text { mento de saúde sobre o perfil } \\
\text { de risco cardiometabólico em } \\
\text { mulheres com síndrome do } \\
\text { ovário policístico: um ensaio } \\
\text { clínico randomizado. }\end{array}$ & $\begin{array}{l}\text { Ensaio clínico } \\
\text { randomizados. }\end{array}$ & 95 & $\begin{array}{l}\text { Dieta baseada em leguminosas } \\
\text { vs. Dieta de mudanças tera- } \\
\text { pêuticas no estilo de vida } \\
\text { (TLC). }\end{array}$ & $\begin{array}{l}\text { O grupo com base nas legu- } \\
\text { minosas teve uma redução } \\
\text { maior na área total sob a } \\
\text { curva para a resposta da insu- } \\
\text { lina (AUC) a um teste de } \\
\text { tolerância oral à glicose e um } \\
\text { maior aumento em HDL-C do } \\
\text { que o grupo TLC. }\end{array}$ \\
\hline $\begin{array}{l}\text { HAIDARI et al., } \\
2020\end{array}$ & $\begin{array}{l}\text { Os efeitos da suplementação de } \\
\text { linhaça no estado metabólico } \\
\text { em mulheres com síndrome dos } \\
\text { ovários policísticos: um ensaio } \\
\text { clínico randomizado e contro- } \\
\text { lado. }\end{array}$ & $\begin{array}{l}\text { Ensaio clínico- } \\
\text { Randomizado. }\end{array}$ & 41 & $\begin{array}{l}\text { Suplementação de pó de linha- } \\
\text { ça ( } 30 \mathrm{~g} / \text { dia) mais modificação } \\
\text { do estilo de vida. }\end{array}$ & $\begin{array}{l}\text { Redução significativa no peso } \\
\text { corporal, concentração de } \\
\text { insulina, avaliação do modelo } \\
\text { homeostático de resistência à } \\
\text { insulina (HOMA-IR), triglice- } \\
\text { rídeos (TG), proteína C reati- } \\
\text { va de alta sensibilidade (hs- } \\
\text { CRP) e leptina. }\end{array}$ \\
\hline $\begin{array}{l}\text { GANIE et al., } \\
2019\end{array}$ & $\begin{array}{l}\text { Avaliação comparativa de } \\
\text { biomarcadores de inflamação } \\
\text { entre mulheres indianas com } \\
\text { síndrome dos ovários policísti- } \\
\text { cos (SOP) que consomem dieta } \\
\text { vegetariana vs. dieta não vege- } \\
\text { tariana. }\end{array}$ & $\begin{array}{l}\text { Estudo observaci- } \\
\text { onal caso contro- } \\
\text { le. }\end{array}$ & 463 & $\begin{array}{l}\text { Dieta vegetariana vs. Dieta } \\
\text { não-vegetariana. }\end{array}$ & $\begin{array}{l}\text { Os marcadores pró- } \\
\text { inflamatórios (hs-CRP sérica, } \\
\text { TNF- } \alpha \text {, IL-6 e IL-1 } \beta \text { ) estavam } \\
\text { elevados e os antiinflamató- } \\
\text { rios (adiponectina sérica e IL- } \\
\text { 10) estavam mais baixos entre } \\
\text { mulheres vegetarianas com } \\
\text { SOP em comparação com } \\
\text { mulheres não vegetarianas } \\
\text { com SOP. }\end{array}$ \\
\hline
\end{tabular}




\begin{tabular}{|c|c|c|c|c|c|}
\hline $\begin{array}{l}\text { BARREA et al., } \\
2019\end{array}$ & $\begin{array}{l}\text { Adesão à Dieta Mediterrânea, } \\
\text { Padrões Alimentares e Compo- } \\
\text { sição Corporal em Mulheres } \\
\text { com Síndrome dos Ovários } \\
\text { Policísticos (SOP). }\end{array}$ & $\begin{array}{l}\text { Estudo transversal } \\
\text { e observacional. }\end{array}$ & 112 & Dieta Mediterrânea (DM). & $\begin{array}{l}\text { Mulheres com SOP consumi- } \\
\text { ram uma quantidade maior de } \\
\text { carboidratos de alto índice } \\
\text { glicêmico, menor quantidade } \\
\text { de fibras, MUFA e PUFA n-3 } \\
\text { em comparação com o grupo } \\
\text { de controle. }\end{array}$ \\
\hline $\begin{array}{l}\text { KAZEMI et al., } \\
2021\end{array}$ & $\begin{array}{l}\text { Ensaio clínico duplo-cego } \\
\text { randomizado que avaliou os } \\
\text { efeitos do ácido elágico na } \\
\text { resistência à insulina, estresse } \\
\text { oxidativo e níveis de hormô- } \\
\text { nios sexuais em mulheres com } \\
\text { síndrome do ovário policístico. }\end{array}$ & $\begin{array}{lr}\text { Ensaio rínico } \\
\text { duplo-cego } \\
\text { domizado. }\end{array}$ & 60 & $\begin{array}{l}\text { Suplementação de } \text { Ácido } \\
\text { Elágico (EA). }\end{array}$ & $\begin{array}{l}\text { A suplementação com EA } \\
\text { diminuiu a glicemia em je- } \\
\text { jum, a insulina e a resistência } \\
\text { à insulina, mostrando uma } \\
\text { mudança significativa do } \\
\text { início do estudo. }(p<0,05) \text {. }\end{array}$ \\
\hline $\begin{array}{l}\text { Kazemi et al., } \\
2021\end{array}$ & $\begin{array}{l}\text { Efeitos do índice glicêmico } \\
\text { dietético e da carga glicêmica } \\
\text { nos perfis cardiometabólico e } \\
\text { reprodutivo em mulheres com } \\
\text { síndrome do ovário policístico: } \\
\text { uma revisão sistemática e meta- } \\
\text { análise de ensaios clínicos } \\
\text { randomizados. }\end{array}$ & $\begin{array}{l}\text { Revisão Sistemá- } \\
\text { tica e meta-análise } \\
\text { de ECRs. }\end{array}$ & 403 & $\begin{array}{l}\text { Dietas com baixo Índice gli- } \\
\text { cêmico (IG) vs. Dietas com } \\
\text { alto IG. }\end{array}$ & $\begin{array}{l}\text { As dietas LGI diminuíram o } \\
\text { HOMA-IR quando compara- } \\
\text { das com as dietas HGI. O } \\
\text { HOMA-IR foi diminuído } \\
\text { apenas em ensaios que aplica- } \\
\text { ram restrição de energia. Não } \\
\text { mostrou diferenças entre as } \\
\text { dietas LGI e HGI nas concen- } \\
\text { trações de glicose em jejum. }\end{array}$ \\
\hline $\begin{array}{l}\text { Alizadeh et al., } \\
2021\end{array}$ & $\begin{array}{l}\text { Efeitos metabólicos e hormo- } \\
\text { nais da suplementação de mela- } \\
\text { tonina e / ou magnésio em } \\
\text { mulheres com síndrome dos } \\
\text { ovários policísticos: um ensaio } \\
\text { randomizado, duplo-cego e } \\
\text { controlado por placebo. }\end{array}$ & $\begin{array}{l}\text { Ensaio randomi- } \\
\text { zado duplo-cego } \\
\text { controlado por } \\
\text { placebo. }\end{array}$ & 84 & $\begin{array}{l}\text { Suplementação de Melatonina, } \\
\text { Magnésio e Melatonina mais } \\
\text { magnésio. }\end{array}$ & $\begin{array}{l}\text { A suplementação de magnésio } \\
\text { em combinação com melato- } \\
\text { nina resultou em uma redução } \\
\text { significativamente maior nas } \\
\text { concentrações de testosterona } \\
\text { em comparação com o place- } \\
\text { bo }(P<0,05) \text {. }\end{array}$ \\
\hline $\begin{array}{l}\text { MIAO et al., } \\
2021\end{array}$ & $\begin{array}{l}\text { Efeitos da suplementação pro- } \\
\text { biótica e simbiótica na resistên- } \\
\text { cia à insulina em mulheres com } \\
\text { síndrome dos ovários policísti- } \\
\text { cos: uma meta-análise. }\end{array}$ & Meta-análise. & 486 & Suplementação de Probióticos. & $\begin{array}{l}\text { A suplementação de probióti- } \\
\text { cos e simbióticos pareceu } \\
\text { melhorar os níveis de avalia- } \\
\text { ção do modelo homeostático } \\
\text { de resistência à insulina e } \\
\text { insulina sérica. }\end{array}$ \\
\hline $\begin{array}{l}\text { SAADATI et al., } \\
2021\end{array}$ & $\begin{array}{l}\text { O efeito da dieta de baixo índi- } \\
\text { ce glicêmico no perfil reprodu- } \\
\text { tivo e clínico de mulheres com } \\
\text { síndrome do ovário policístico: } \\
\text { uma revisão sistemática e meta- } \\
\text { análise. }\end{array}$ & $\begin{array}{lr}\text { Revisão } & \text { Sistemá- } \\
\text { tica e } & \text { meta- } \\
\text { análise. }\end{array}$ & 412 & $\begin{array}{l}\text { Dietas com baixo índice gli- } \\
\text { cêmico (LGD). }\end{array}$ & $\begin{array}{l}\text { Saúde emocional medida e } \\
\text { pelos corporais foram signifi- } \\
\text { cativamente mais baixos em } \\
\text { mulheres em grupo de Dietas } \\
\text { com baixo índice glicêmico. }\end{array}$ \\
\hline
\end{tabular}

Fonte: Dados da pesquisa (2021).

A investigação de Zhang et al., (2019), buscou examinar a eficácia de uma dieta com ingestão de $45 \%$ ou menos de carboidratos em melhorar os sintomas clínicos associados à SOP, em comparação com dietas tradicionais ou ricas em carboidratos. Foi encontrado que tal intervenção pode reduzir consideravelmente o IMC e os níveis séricos de colesterol total (CT) e LDL-C nessa população clínica, e que, em particular, LCD (low carb diet) associada a um baixo teor de gordura $(<35 \%)$ e de longo prazo (>4semanas) podem aumentar significativamente os níveis de FSH (hormônio folículo estimulante) e SHBG (globulina ligadora de hormônios sexuais) bem como, diminuir o nível de testosterona em pacientes com SOP.

No entanto, é crucial reconhecer que uma redução na quantidade de ingestão de carboidratos por si só pode não ser ideal ou uma estratégia prática para todas as mulheres com SOP. Foi avaliado o efeito de uma dieta baseada em leguminosas e portanto, de baixo IG, no perfil metabólico de mulheres com SOP, que mostrou a eficácia da mesma na redução da AUC - área sob a curva total de insulina, níveis de LDL-C, TG (triglicérides), relação HDL/CT, pressão arterial diastólica e aumento da concentração de HDL-C. Sementes comestíveis secas da família das leguminosas, incluindo ervilhas, feijões, lentilhas e grão de bico, são ricas em fibras, contêm carboidratos complexos com baixo IG, são pobres em gordura, contêm proteínas de alta qualidade, baixo teor de sódio e são uma fonte significativa de vitaminas e minerais, como ferro, zinco, folato, cálcio, magnésio e potássio. Seus efeitos hipocolesterolêmicos são multifatoriais e podem ser atribuídos principalmente ao seu alto teor de fibras (Kazemi et al., 2018). 
Para Saadati et al., (2021) pacientes com SOP, devem seguir dietas especiais, como de baixo índice glicêmico, com efeitos potencialmente positivos na glicose sanguínea e na insulina, que podem reduzir o risco da doença e seus eventos adversos associados. Dietas ricas em açúcar estão associadas a riscos aumentados de resistência à insulina e dislipidemia, fatores de risco estabelecidos para distúrbios ovulatórios. (Wilis et al., 2020)

A revisão sistemática e meta-análise de 10 ensaio clínicos randomizados (ECRS), realizado com mulheres com SOP, mostrou que dietas de baixo índice glicêmico em comparação com as dietas de alto índice glicêmico melhoraram 1) o estado glicorregulatório avaliado pela diminuição do HOMA-IR e insulina em jejum; 2) perfil lipídico, evidenciado pela redução do CT, colesterol LDL e TGs ; 3) adiposidade abdominal, evidenciada pela diminuição da CC; e 4) hiperandrogenismo, conforme avaliado pela redução da testosterona total (Kazemi et al., 2021).

Outra intervenção nutricional analisada no presente estudo foi o padrão vegetariano e não vegetariano em mulheres com SOP de dois centros diferentes no norte da Índia, no qual, curiosamente as mulheres vegetarianas apresentaram níveis mais elevados de pró-inflamatórios séricos (TNF- $\alpha$, IL-6, hs-CRP, resistina e IL-1 $\beta$ ) e níveis mais baixos de marcadores antiinflamatórios (IL-10 sérica e adiponectina). Também há sugestão de que a adesão estrita às dietas vegetarianas sem a suplementação necessária afeta adversamente os resultados da fertilidade em mulheres (Ganie et al., 2019).

No entanto, há evidências de que a adesão a dietas constituída em sua maioria por alimentos de origem vegetal, como a DM - Dieta Mediterrânea, está inversamente associada à adiposidade, RI, risco de diabetes mellitus tipo 2 e doença cardiovascular. É considerado o potencial nutracêutico de vários alimentos da dieta mediterrânea, inclusive o azeite de oliva extra-virgem, que é um dos principais componentes desse padrão alimentar. Paralelamente aos carboidratos, a gordura dietética e, em particular, os MUFA e os PUFA n-3, também estão implicados na patogênese da SOP. Uma meta-análise recente de nove estudos envolvendo 591 indivíduos encontrou que a suplementação de n-3 PUFA deve ser recomendada para o controle metabólico de mulheres com SOP (Barrea et al., 2019)

Alguns pesquisadores sugeriram que os ácidos graxos ômega-3 na semente de linhaça podem aumentar o nível de adiponectina, que tem propriedades antiateroscleróticas, antidiabéticas e antiinflamatórias, melhorando a sensibilidade à insulina nas pacientes com SOP (Haidari et al., 2020)

O uso de compostos antioxidantes na redução de RI e inflamação crônica e, consequentemente, um melhor gerenciamento da síndrome de SOP também tem sido de particular interesse em pesquisas recentes. Os polifenóis como metabólitos vegetais secundários são encontrados em vegetais e frutas. Como antioxidantes potentes, eles têm efeitos protetores e terapêuticos no controle dos efeitos do estresse oxidativo, regulando citocinas e enzimas inflamatórias, aumentando a defesa antioxidante e suprimindo as vias inflamatórias e seus mecanismos de sinalização celular. $\mathrm{O}$ ácido elágico, um polifenol encontrado naturalmente em várias frutas e vegetais, incluindo morangos, framboesas vermelhas, romãs e uvas, demonstrou ter um efeito significativo em enzimas importantes no metabolismo do colesterol (Kazemi et al., 2021).

Visto que a resistência à insulina é uma importante característica etiológica da SOP, e que as mulheres afetadas têm maior risco de desenvolver diabetes tipo 2 e complicações metabólicas relacionadas, foi relatado que os distúrbios do sono são comuns na SOP, e algumas formas deles, como a apneia obstrutiva do sono, exacerba a resistência à insulina. Como a melatonina é conhecida por ser um regulador eficaz do ritmo circadiano e promover o sono, os efeitos benéficos na qualidade do sono são de se esperar. Em vários estudos, foi demonstrado que a excreção urinária de 6-sulfatoximelatonina, o principal metabólito excretor da melatonina, junto com os níveis de melatonina no sangue e na saliva foi maior em pacientes com SOP do que em mulheres com fertilidade normal e os níveis dessas moléculas (6-sulfatoximelatonina urinária e melatonina sérica) foram significativamente correlacionadas com a gravidade dos distúrbios do sono (Alizadeh et al., 2021).

Além disso, mulheres com SOP relatam maior sonolência diurna excessiva e redução da qualidade do sono em comparação com indivíduos controle. Níveis elevados de melatonina noturno e matinal e menor variabilidade dia-noite nas 
concentrações de melatonina foram relatados em mulheres com SOP em comparação com seus pares saudáveis (Simon et al., 2019)

A resistência à insulina está intimamente relacionada a microbiota intestinal, portanto mudanças dinâmicas nessa estrutura leva à ocorrência e o desenvolvimento de uma variedade de doenças metabólicas endócrinas. Estudos recentes demonstraram que a suplementação de probióticos e simbióticos afeta o estado metabólico da RI. Os mecanismos pelos quais a microbiota intestinal melhora a RI incluem a redução da permeabilidade intestinal por meio da manutenção da função de barreira epitelial e redução da inflamação via lipopolissacarídeo ou ácidos graxos de cadeia curta (Miao et al., 2021). Uma composição de probióticos e prebióticos que aumentam a viabilidade dos probióticos no trato intestinal, estimulando o crescimento ou melhorando a atividade metabólica, é chamada de simbiótica (Darvishi et al., 2021)

Os sintomas da SOP e o risco geral de desenvolver problemas de saúde a longo prazo podem ser melhorados muito com a perda do excesso de peso. A composição da dieta também é importante, conforme relatado acima e modificações dietéticas são recomendadas para melhorar o estado metabólico. No entanto, as mulheres com SOP geralmente não aderem às recomendações dietéticas de longo prazo, com escassa melhora nos resultados metabólicos e reprodutivos (Calcaterra et al., 2021)

Estudos que objetivam identificar as intervenções dietéticas, em comparação com nenhuma dieta ou dietas diferentes, como meio eficazes para melhorar os resultados de perda de peso, metabolismo, fertilidade e bem-estar emocional em mulheres com SOP, sugerem que, para pessoas com excesso de peso, um déficit de energia de 30\% ou 500-750 kcal/dia (1200 a $1.500 \mathrm{kcal} / \mathrm{dia}$ ) podem ser prescritos para mulheres com SOP, sempre considerando as necessidades energéticas individuais, o peso corporal e os níveis de atividade física bem como, as preferências alimentares, necessidades culturais e uma abordagem flexível e individual. A composição de uma dieta específica em intervenções no estilo de vida permanece controversa visto as recomendações gerais para reduzir a ingestão calórica (energia), em vez de modificar a composição dos macronutriente. (Diretriz Internacional SOP, 2018).

\section{Considerações Finais}

O presente estudo se alinha com as evidências de que uma variedade de condutas dietéticas balanceadas, como dietas com alimentos de baixo índice glicêmico, suplementação de ômega 3, aumento da ingestão de leguminosas e compostos fenólicos, podem e devem ser recomendados para o manejo das manifestações clínicas e metabólicas em mulheres com SOP. Além disso, os princípios gerais de uma alimentação saudável, de acordo com as recomendações para a população geral, são cabíveis para todas as mulheres com SOP. Para estas, não há ou há evidências limitadas de que qualquer tipo específico de dieta é melhor do que outro. Estudos mais rigorosos, com uma amostra significativa, são necessários para assegurar a eficácia da dieta no manejo da SOP.

\section{Referências}

Barrea, L., Arnone, A., Annunziata, G., Muscogiuri, G., Laudisio, D., Salzano, C., Pugliese, G., Colao, A., \& Savastano, S. (2019). Adherence to the Mediterranean Diet, Dietary Patterns and Body Composition in Women with Polycystic Ovary Syndrome (PCOS). Nutrients, 11(10), 2278. https://doi.org/10.3390/nu11102278

Calcaterra, V., Verduci, E., Cena, H., Magenes, V. C., Todisco, C. F., Tenuta, E., Gregorio, C., de Giuseppe, R., Bosetti, A., di Profio, E., \& Zuccotti, G. (2021). Polycystic Ovary Syndrome in Insulin-Resistant Adolescents with Obesity: The Role of Nutrition Therapy and Food Supplements as a Strategy to Protect Fertility. Nutrients, 13(6), 1848. https://doi.org/10.3390/nu13061848

Darvishi, S., Rafraf, M., Asghari-Jafarabadi, M., \& Farzadi, L. (2021). Synbiotic Supplementation Improves Metabolic Factors and Obesity Values in Women with Polycystic Ovary Syndrome Independent of Affecting Apelin Levels: A Randomized Double-Blind Placebo - Controlled Clinical Trial. International Journal of Fertility \& Sterility, 15(1), 51-59. https://doi.org/10.22074/ijfs.2021.6186 
Ganie, M. A., Sahar, T., Rashid, A., Wani, I. A., Nisar, S., Sathyapolan, T., Vishnubhatla, S., Ramakrishnan, L., Parvez, T., \& Geer, I. (2019). Avaliação comparativa de biomarcadores de inflamação entre mulheres indianas com síndrome dos ovários policísticos (SOP) que consomem dieta vegetariana vs. dieta não vegetariana. Front Endocrinol (Lausanne), 10(2019), 10-699

Haidari, F., Banaei-Jahromi, N., Zakerkish, M., \& Ahmadi, K. (2020). The effects of flaxseed supplementation on metabolic status in women with polycystic ovary syndrome: a randomized open-labeled controlled clinical trial. Nutrition Journal, 19(1), 8. https://doi.org/10.1186/s12937-020-0524-5

Hallajzadeh, J., Khoramdad, M., Karamzad, N., Almasi-Hashiani, A., Janati, A., Ayubi, E., Pakzad, R., Sullman, M. J. M., \& Safiri, S. (2018). Metabolic syndrome and its components among women with polycystic ovary syndrome: a systematic review and meta-analysis. Journal of Cardiovascular and Thoracic Research, 10(2), 56-69. https://doi.org/10.15171/jcvtr.2018.10

Kazemi, M., Hadi, A., Pierson, R. A., Lujan, M. E., Zello, G. A., \& Chilibeck, P. D. (2021). Effects of Dietary Glycemic Index and Glycemic Load on Cardiometabolic and Reproductive Profiles in Women with Polycystic Oçvary Syndrome: A Systematic Review and Meta-analysis of Randomized Controlled Trials. Advances in Nutrition (Bethesda, Md.), 12(1), 161-178. https://doi.org/10.1093/advances/nmaa092

Kazemi, M., Lalooha, F., Nooshabadi, M. R., Dashti, F., Kavianpour, M., \& Haghighian, H. K. (2021). Randomized double blind clinical trial evaluating the Ellagic acid effects on insulin resistance, oxidative stress and sex hormones levels in women with polycystic ovarian syndrome. Journal of Ovarian Research, 14(1). https://doi.org/10.1186/s13048-021-00849-2

Kazemi, M., McBreairty, L. E., Chizen, D. R., Pierson, R. A., Chilibeck, P. D., \& Zello, G. A. (2018). A Comparison of a Pulse-Based Diet and the Therapeutic Lifestyle Changes Diet in Combination with Exercise and Health Counselling on the Cardio-Metabolic Risk Profile in Women with Polycystic Ovary Syndrome: A Randomized Controlled Trial. Nutrients, 10(10), 1387. https://doi.org/10.3390/nu10101387

Kite, C., Lahart, I. M., Afzal, I., Broom, D. R., Randeva, H., Kyrou, I., \& Brown, J. E. (2019). Exercise, or exercise and diet for the management of polycystic ovary syndrome: a systematic review and meta-analysis. Systematic Reviews, 8(1), 51. https://doi.org/10.1186/s13643-019-0962-3

Moran, L. J., Noakes, M., Clifton, P., Buckley, J., Brinkworth, G., Thomson, R., \& Norman, R. J. (2019). Predictors of Lifestyle Intervention Attrition or Weight Loss Success in Women with Polycystic Ovary Syndrome Who Are Overweight or Obese. Nutrients, 11(3), 492. https://doi.org/10.3390/nu11030492

Saadati, N., Haidari, F., Barati, M., Nikbakht, R., Mirmomeni, G., \& Rahim, F. (2021). The effect of low glycemic index diet on the reproductive and clinical profile in women with polycystic ovarian syndrome: A systematic review and meta-analysis. Heliyon, 7(11), e08338. https://doi.org/10.1016/j.heliyon.2021.e08338

Salek, M., Clark, C. C. T., Taghizadeh, M., \& Jafarnejad, S. (2019). N-3 fatty acids as preventive and therapeutic agents in attenuating PCOS complications. EXCLI Journal, 18, 558-575. https://doi.org/10.17179/excli2019-1534

Shang, Y., Zhou, H., He, R., \& Lu, W. (2021). Dietary Modification for Reproductive Health in Women With Polycystic Ovary Syndrome: A Systematic Review and Meta-Analysis. Frontiers in Endocrinology, 12, 735954. https://doi.org/10.3389/fendo.2021.735954

Simon, S. L., McWhirter, L., Diniz Behn, C., Bubar, K. M., Kaar, J. L., Pyle, L., Rahat, H., Garcia-Reyes, Y., Carreau, A.-M., Wright, K. P., Nadeau, K. J., \& Cree-Green, M. (2019). Morning Circadian Misalignment Is Associated With Insulin Resistance in Girls With Obesity and Polycystic Ovarian Syndrome. The Journal of Clinical Endocrinology and Metabolism, 104(8), 3525-3534. https://doi.org/10.1210/jc.2018-02385

Síndrome dos ovários policísticos. São Paulo: Federação Brasileira das Associações de Ginecologia e Obstetrícia (FEBRASGO). 2018. 103p. (Série Orientações e Recomendações FEBRASGO, n.4, Comissão Nacional de Ginecologia Endócrina)

Willis, S. K., Wise, L. A., Wesselink, A. K., Rothman, K. J., Mikkelsen, E. M., Tucker, K. L., Trolle, E., \& Hatch, E. E. (2020). Glycemic load, dietary fiber, and added sugar and fecundability in 2 preconception cohorts. The American Journal of Clinical Nutrition, 112(1), 27-38. https://doi.org/10.1093/ajcn/nqz312

Yonekura, T., Quintans, J. R., Soares, C. B., \& Negri Filho, A. A. de. (2019). Revisão realista como metodologia para utilização de evidências em políticas de saúde: uma revisão integrativa. Revista Da Escola de Enfermagem Da USP, 53. https://doi.org/10.1590/s1980-220x2018037703515

Zhang, X., Zheng, Y., Guo, Y., \& Lai, Z. (2019). The Effect of Low Carbohydrate Diet on Polycystic Ovary Syndrome: A Meta-Analysis of Randomized Controlled Trials. International Journal of Endocrinology, 2019, 4386401. https://doi.org/10.1155/2019/4386401

Zhou, L., Ni, Z., Cheng, W., Yu, J., Sun, S., Zhai, D., Yu, C., \& Cai, Z. (2020). Characteristic gut microbiota and predicted metabolic functions in women with PCOS. Endocrine Connections, 9(1), 63-73. https://doi.org/10.1530/EC-19-0522 